\title{
Postpartum Haemorrhage in the Developing World A Review of Clinical Management Strategies
}

\author{
John W. Snelgrove*
}

\begin{abstract}
The developing world is disproportionately burdened with high rates of maternal mortality. Despite widespread reduction in maternal deaths due to improved antepartum, intrapartum, and postpartum care in developed nations, mortality rates are persistently high in many countries unable to provide advanced medical care. Postpartum haemorrhage accounts for a substantial proportion of maternal deaths in developing countries. This review addresses the clinical management strategies aimed at the prevention and treatment of postpartum haemorrhage that are effective in lowresource settings. The review was conducted by searching the English-language medical literature using MEDLINE (1950 - Feb 2009) and the online search engine Google Scholar. Four relevant strategies were identified in the literature: active management of the third stage of labour (AMSTL), the use of uterotonic agents including misoprostol, accurate measurement of blood loss, and internal and external compression techniques. Despite known intervention options, additional research on effective management strategies and their implementation is needed to address postpartum haemorrhage in countries of the developing world.
\end{abstract}

\section{INTRODUCTION}

Healthy mothers are the focus of United Nations' Fifth Millennium Development Goal, with the aim of lowering maternal mortality ratios by $75 \%$ between 1990 and 2015 (1). Despite reduction in maternal deaths amongst transitional and developed countries, maternal mortality remains a significant cause of death for women of reproductive age in the developing world. This fact is exemplified by statistics collected by the WHO, which show that $99 \%$ of the world's half-million annual maternal deaths occur in developing countries (2). Lifetime risk of maternal death is as high as one in six for women living in the world's poorest nations, a figure in sharp contrast to a risk of one in 30,000 for women in Northern Europe (3). Substantial progress is required before the targets of Goal No. 5 are met, and increasingly this progress must be seen within the

*To whom correspondence should be addressed:

John W. Snelgrove

807-205 Oxford St. E.

London, ON

N6A 5G5

jsnelgrove2011@meds.uwo.ca nations disproportionately burdened by maternal deaths.

Maternal mortality can be conceptualised as a direct result of obstetrical complication leading to death, or indirectly from a previously existing disease for which pregnancy exacerbates the pathology or contributes to increased severity of illness. Direct causes account for the great majority of maternal deaths in the developing world (3). A systematic review conducted by the WHO found that postpartum haemorrhage is the leading cause of maternal mortality in Africa and Asia, accounting for up to half of the total number of deaths in these regions (4). Overall, postpartum haemorrhage accounts for an estimated $25 \%$ of maternal mortality worldwide (2). Hypertensive disorders such as eclampsia were identified as the leading causes of death in Latin America and the Caribbean; other prevalent causes of maternal mortality include sepsis, obstructed labour, and complications with abortion (2).

This review addresses the question of which clinical management strategies are effective in treating postpartum haemorrhage in the developing world. Relevance of management options to the low-resource 
settings characteristic of developing countries is especially considered. While equally important health infrastructure and policy interventions are necessary to decrease maternal mortality in developing countries $(2,3)$, these are not the focus of this review.

\section{METHODS}

A search of the available English-language medical literature was undertaken using MEDLINE (1950-Feb. 2009) and an online search engine, Google Scholar. Terms used in searches included combinations of "maternal mortality", "postpartum haemorrhage/ hemorrhage", "labor/labour complications", and "developing countries." Abstracts from potentially relevant publications were read for content and included in the review if they addressed the clinical management of postpartum haemorrhage in a developing country or low-resource setting.

\section{CLINICAL MANAGEMENT OF POSTPARTUM HAEMORRHAGE}

Postpartum haemorrhage is defined as blood loss of more than $500 \mathrm{~mL}$ within 24 hours of vaginal delivery. Blood loss may be associated with retained or adherent placenta, trauma to tissue and vessels during delivery, uterine atony, or coagulopathies such as fibrinolysis and afibrinogenaemia (5). The known risk factors for postpartum haemorrhage include prolonged third stage of labour, multiple delivery, fetal macrosomia, episiotomy, and previous history of postpartum haemorrhage $(6,7)$. Postpartum haemorrhage may have a predilection toward certain ethnicities; however, this is not a consistent finding. In a low-resource setting, advanced maternal age and low parity have been shown to associate with a higher risk of haemorrhage (8). Importantly, postpartum haemorrhage may occur in obstetrical patients with an absence of known risk factors $(3,8)$.

The clinical management of postpartum haemorrhage has not been systematically assessed with respect to the reduction of maternal mortality in the context of developing countries. Indeed, maternal mortality has been used as a primary outcome in only a few randomised controlled trials, largely because of the unethical nature of withholding care that is obviously life-saving, but also as a result of the necessary sample size requirements to show an effect on mortality (9). Fortunately, clinical management strategies to prevent or reduce blood loss due to postpartum haemorrhage have been more broadly considered in the literature (927). This review identified four management strategies relevant to the topic of postpartum haemorrhage in developing countries: the active management of the third stage of labour, the use of uterotonic agents, early and accurate blood loss estimation, and compression techniques to reduce blood loss.

\section{Active Management of the Third Stage of Labour}

Active management of the third stage of labour (AMTSL) is comprised of therapies and pharmacological treatment directed at reducing the risk of postpartum haemorrhage. This management strategy is in contrast to "expectant" management of the third stage of labour, whereby therapeutic intervention to aid in the expulsion of the placenta is not performed. Modalities of treatment in AMTSL include the administration of a uterotonic agent, umbilical cord traction, fundal massage, and may or may not include early cord clamping (10). A large randomised controlled trial found evidence that AMSTL reduced the odds of postpartum haemorrhage by half compared to non-active delivery of the placenta $(\mathrm{OR}=0.50,95 \% \mathrm{CI}$ 0.34-0.73) (11). These findings were reflected in a retrospective cohort study with historical controls conducted in a developing country, which found a significant reduction in incidence of postpartum haemorrhage after implementation of AMSTL management (10). The evidence is in favour of AMTSL as a relatively inexpensive and effective management modality for controlling postpartum haemorrhage in developing countries, providing the technique is performed appropriately. Evidence from a Cochrane Review (12) on active versus expectant management is consistent with this recommendation: significantly reduced odds of postpartum haemorrhage were observed with the use of AMSTL in the meta-analysis of Khan and colleagues (11), which focussed on the developing world. Side effects of nausea, vomiting, and raised blood pressure were more strongly associated with AMSTL than expectant management, though these were considered less adverse than the higher risk of postpartum haemorrhage found with expectant management (10-12).

\section{Misoprostol as a Uterotonic Agent}

Uterotonic agents are administered during active management of the third stage of labour to promote uterine contractions and the expulsion of placental tissue $(10,12,13,15,19)$. Regarding the appropriate choice of uterotonic agent, oxytocin is favoured for the medical management of postpartum haemorrhage, based on a large, multicentre randomised controlled trial conducted by the WHO (13). This study showed that oxytocin is associated with significantly less risk of haemorrhage (3\% versus $4 \%$ incidence in the oxytocin and misoprostol groups respectively, $\mathrm{p}<0.001$ ) and fewer side effects than misoprostol (13). This result is consistent with a Cochrane systematic review, which 
assessed 37 trials from both developed and developing nations and concluded that oxytocin is more effective than misoprostol against postpartum haemorrhage and associated sequelae (14). Smaller randomised controlled trials conducted specifically in developing countries found significant evidence of reduced postpartum haemorrhage risk with misoprostol compared to placebo $(15,16)$, and some reported no difference when compared to oxytocin $(17,18)$. The above studies concluded that misoprostol is effective as a uterotonic agent to prevent and treat postpartum haemorrhage.

The choice of uterotonic agent for treatment of postpartum haemorrhage in developing countries must be considered in the context of both expense and thermostability. Costly or heat-labile preparations requiring refrigeration may be difficult or impossible to use in low-resource settings $(17,18)$. Misoprostol is a relatively inexpensive, thermostable uterotonic agent which can be administered orally, rectally, or vaginally (16-19). This makes it a likely candidate for effective medical prevention and management of postpartum haemorrhage in the developing world despite the therapeutic advantage provided by oxytocin, which is more expensive, heat-labile, and requires IV or IM administration (19). Given that oxytocin may provide an advantage over misoprostol and is associated with fewer side-effects, its use in areas with refrigeration capability and adequate financing is favoured over misoprostol in settings where AMSTL is the norm $(13,14,19)$. However, in low-resource settings, misoprostol should be considered as an alternative primary treatment for postpartum haemorrhage.

\section{Early and Accurate Estimation of Blood Loss}

Not surprisingly, the severity of clinical outcomes for postpartum haemorrhage correlates with the amount of blood lost, and for this reason, the early and accurate estimation of blood loss is vitally important to the clinical management of the postpartum bleeding patient (20). Low-resource settings may not have access to standardised blood collection tools, however, novel approaches developed to estimate blood loss may provide effective alternatives. For example, Patel and colleagues describe the effectiveness of an inexpensive blood collection drape used in India to estimate blood loss against visual assessment alone. Estimates based on the drape correlated well with photospectrometry and were more accurate than visual assessment alone, which tended to underestimate the amount of blood lost (21). This suggests the tool provides an appropriate measurement that can be easily and inexpensively made in low-resource settings. Geller and colleagues indicate that this system is now used in eight countries (22). A similar collection system used in Tanzania provides a consistent measure of blood loss with the use of kanga, a type of blanket with standard dimensions that is used regionally in East Africa and holds approximately 250 $\mathrm{mL}$ of blood when soaked (23). The effectiveness of blood loss estimation using these techniques has not been established empirically with respect to postpartum haemorrhage outcomes. However, standardised, accurate measurement tools would theoretically allow birth attendants to more easily recognise postpartum haemorrhage in a systematic way, resulting in opportunities for intervention and appropriate management. Although these approaches have been described in only a handful of studies, they demonstrate the importance of solutions that are regionally acceptable and relevant - such as calibrated measures in Tanzania based on the amount of blood absorbed by a kanga.

\section{External and Internal Compression}

Blood loss resulting from postpartum haemorrhage may be managed with the use of compression techniques suited or adapted to use in low-resource settings. Abdominal compression of the aorta may decrease blood loss in severe postpartum haemorrhage if performed correctly (24). This manoeuvre is easy to perform and requires little equipment, thus it would present fewer barriers to implementation in developing countries than many other therapies. However, there is a dearth of evidence showing that abdominal compression of the aorta significantly reduces negative outcomes associated with postpartum haemorrhage specifically. Intrauterine tamponade with the use of a condom and rubber catheter has been suggested as an affordable alternative to blood loss management in severe postpartum haemorrhage refractory to uterotonic therapy. The evidence for this technique is based on several series of case reports, the largest of which involved 23 obstetric patients and was conducted in Bangladesh (25).

\section{DISCUSSION}

The proper management of postpartum haemorrhage is an essential component of obstetric care and a necessary step in the goal to reduce worldwide maternal mortality. The literature is replete with evidence for best practices and appropriate therapies, particularly with respect to uterotonic agents and active management of the third stage of labour. However, the interventions predominantly identified in the research and clinical settings of developed countries may not be feasible in the low-resource settings characteristic of the developing world $(9,22,26)$. Strategies that prevent and treat postpartum haemorrhage must be readily 
accessible, affordable, and have uncomplicated storage requirements in order to be effective in countries with limited healthcare financing and infrastructure. A ready example of this phenomenon is the suggested use of misoprostol as a uterotonic agent in the absence of resources that make the provision of the superior therapy (oxytocin) possible. An urgent need for the uptake of technologies proven to work in developing countries has been identified. Furthermore, continued research into the effectiveness of postpartum haemorrhage interventions in the context of lowresource settings is required to better understand the clinical management of this obstetrical complication. These needs extend beyond postpartum haemorrhage to include the other direct and indirect causes of maternal mortality in the developing world. Contextually appropriate strategies to manage hypertensive disorders (e.g. eclampsia), sepsis, obstructed labour, and complications related to abortion have been identified but still require implementation to be effective against maternal mortality $(9,26)$.

This review identified four clinical management strategies for postpartum haemorrhage, however a fifth clinical consideration needs also to be addressed, although it is not specific to reduced postpartum haemorrhage alone: the presence of skilled birth attendants during delivery. These healthcare workers and midwives possess (at least theoretically) a minimum skill set necessary to take a detailed history, provide antenatal care, perform a vaginal exam, time and assess uterine contractions, and otherwise manage normal labour and recognise the need to refer in the presence of complications (27). The availability of skilled birth attendants during delivery may be an important step toward maternal mortality reduction, as suggested by ecological data from the WHO (28). However, caution must be exercised with recommendations regarding skilled attendance at birth. Intrapartum care is largely dependent on setting, transportation, and the availability of referral networks to manage complications (9). Additionally, the lack of a clear definition regarding the practice scope of skilled birth attendants continues to confuse their role in providing healthcare. Despite these areas of ambiguity, Graham and colleagues note the positive impact attendants may have on reducing maternal mortality, so long as specific skill sets are mastered and adequate access to equipment and referral services is available (27).

In considering only the clinical management of postpartum haemorrhage, this review necessarily takes a narrow view of the causes of maternal mortality. Ronsmans and colleagues describe the other levels at which delays in access to care can prove fatal to mothers experiencing complications with pregnancy and delivery. The authors describe three potential delays to treatment, the first being a delay in the patient's or family's recognition of the need to seek care (3). This can be further explained as discordance between actual and perceived risk, the latter being partly constituted by attitudes and cultural beliefs such as, for example, the benefit or normalcy of bleeding during delivery (29). Added to this "first delay" would be a family's inability to afford care in settings that lack publically funded healthcare. Uncoordinated referral systems and barriers at the community level —including physical, financial, or cultural-comprise the second delay, while delay in receiving effective interventions is cited as the third (3). As mentioned previously, this review focuses on clinical management strategies, akin to the interventions necessary to avoid the "third delay". Nonetheless, the extent to which appropriate management may improve clinical outcomes is limited by factors described by delays "one" and "two". Socioeconomic inequalities predispose women from poorer backgrounds to increased risk of death during childbirth, as does the lower social status women hold in many communities of the developing world $(3,29)$. Additionally, healthcare systems in developing countries may not be able to provide comprehensive care to patients as a result of limited access to resources. In an ecological analysis of Latin American and Caribbean countries, Cruz found an inverse association between donor blood availability and both maternal mortality ratios and risk of death due to postpartum haemorrhage (30). Social and cultural inequalities, along with issues concerning the accessibility and comprehensiveness of healthcare, are the antecedents of maternal mortality in developing nations. These upstream factors need to be considered alongside clinical management and intervention in the pursuit of worldwide reduction in maternal deaths.

\section{SUMMARY OF RECOMMENDATIONS}

This review identified several evidence-based recommendations for the prevention and management of postpartum haemorrhage in developing countries. Broadly, these recommendations relate to AMSTL,the use of uterotonic therapy, the use of compression techniques, and the accurate measurement of blood loss. These recommendations and their level of evidence are summarised below.

Recommendations Based on Level A Evidence

1. AMTSL should be practised by clinicians to reduce risk of postpartum haemorrhage (10-12).

2. Oxytocin should be used as a uterotonic agent in settings where the appropriate storage and administration of this medication is possible $(13,14)$. 
3. In the absence of available oxytocin, misoprostol is an effective uterotonic agent and should be used to help prevent postpartum haemorrhage (17-19).

\section{Recommendations Based on Level B Evidence}

1. Intrauterine tamponade with condom and catheter may be used to control severe postpartum haemorrhage refractory to medical management (25).

\section{Recommendations Based on Level C Evidence}

1. Accurate estimation of blood loss should be standardised using a blood collection drape (21-23).

2. Abdominal compression of the aorta may decrease blood loss in severe postpartum haemorrhage (24).

\section{REFERENCES}

1. United Nations. Millennium Development Goals, Goal 5: improve maternal health. Yerevan (Armenia): UN Department of Public Information; 2008 Sep.

2. World Health Organization. Maternal mortality in 2005: estimates developed by WHO, UNICEF, UNFPA and the World Bank. Geneva (Switzerland): WHO; 2007.

3. Ronsmans C, Graham WJ; Lancet Maternal Survival Series Steering Group. Maternal mortality: who, when, where, and why. Lancet. 2006;368:1189-1200.

4. Khan KS, Wojdyla D, Say L, Gülmezoglu AM, Van Look PFA; WHO analysis of causes of maternal death: a systematic review. Lancet. 2006;367:1066-1074.

5. World Health Organization. International statistical classification of diseases and related health problems, 10th revision (ICD-10). Geneva (Switzerland): WHO; 2007.

6. Coombs CA, Murphy EL, Laros RK Jr. Factors associated with postpartum hemorrhage with vaginal birth. Obstet Gynecol. 1991;77(1):69-76.

7. Magann EF, Evans S, Hutchinson M, Collins R, Howard BC, Morrison JC. Postpartum hemorrhage after vaginal birth: an analysis of risk factors. Southern Med J. 2005;98(4):419-422.

8. Tsu V. Postpartum haemorrhage in Zimbabwe: a risk factor analysis. British J Obstet Gynaecol. 1993;100:327-333.

9. Campbell OMR, Graham WJ; Lancet Maternal Survival Series Steering Group. Strategies for reducing maternal mortality: getting on with what works. Lancet. 2006;368:1284-1299.

10. Geelhoed D, Visser L, Agordzo P, Asare K, Van Leeuwen JS, Van Roosmalen J. Active versus expectant management of the third stage of labor in rural Ghana. Acta Obstet Gynecol Scand. 2002;81:172-173.

11. Khan, GQ, John IS, Wani S, Doherty T, Sibai BM. Controlled cord traction versus minimal intervention techniques in delivery of the placenta: a randomized controlled trial. Am J Obstet Gynecol. 1997;177(4);770-774.

12. Prendiville WJP, Elbourne D, McDonald SJ. Active versus expectant management in the third stage of labour. Cochrane Database of Systematic Reviews. 2000;(3).

13. Gülmezoglu AM, Villar J, Ngoc NTN, Piaggio G, Carroli G, Adetoro L, et al.; WHO Collaborative Group to Evaluate Misoprostol in the Management of the Third Stage of Labour. WHO multicentre randomised trial of misoprostol in the management of the third stage of labour. Lancet. 2001:358;689695.
14. Gülmezoglu AM, Forna F, Villar J, Hofmeyr GJ. Prostaglandins for preventing postpartum haemorrhage. Cochrane Database of Systematic Reviews. 2007;(3).

15. Walraven G, Blum J, Dampha Y, Sowe M, Morison L, Winikoff $\mathrm{B}$, et al. Misoprostol in the management of the third stage of labour in the home delivery setting in rural Gambia: A randomised controlled trial. BJOG: Int J Obstet Gynaecol. 2005;112:1277-1283.

16. Derman RJ, Kodkany BS, Goudar SS, Geller SE, Naik VA, Bellad MB, et al. Oral misoprostol in preventing postpartum haemorrhage in resource-poor communities: a randomised controlled trial. Lancet. 2006;368:1248-1253.

17. Lokugamage AU, Sullivan KR, Niculescu I, Tigere $P$, Onyangunga $\mathrm{F}$, El-Refaey $\mathrm{H}$, et al. A randomized study comparing rectally administered misoprostol versus Syntometrine combined with an oxytocin infusion for the cessation of primary post partum hemorrhage. Acta Obstet Gynecol Scand. 2001;80:835-839.

18. Walley RL, Wilson JB, Crane JMG, Matthews K, Sawyer E, Hutchens D. A double-blind placebo controlled randomised trial of misoprostol and oxytocin in the management of the third stage of labour. British J Obstet Gynaecol. 2000;107;1111-1115.

19. Alfirevic Z, Blum J, Walraven G, Weeks A, Winikoff B. Prevention of postpartum hemorrhage with misoprostol. Int J Gynecol Obstet. 2007;99:S198-S201.

20. Chhabra S, Sirohi R. Trends in maternal mortality due to haemorrhage: two decades of Indian rural observations. J Obstet Gynaecol. 2004;24(1):40-43.

21. Patel A, Goudar SS, Geller SE, Kodkany BS, Edlavitch SA, Wagh K, et al. Drape estimation vs. visual assessment for estimating postpartum hemorrhage. Int $\mathrm{J}$ Gynecol Obstet. 2006;93:220-224.

22. Geller SE, Adams MG, Kelly PJ, Kodkany BS, Derman RJ. Postpartum hemorrhage in resource-poor settings. Int J Gynecol Obstet. 2006;92:202-211.

23. Prata N, Mbaruku G, Campbell M, Potts M, Vahidnia F. Controlling postpartum hemorrhage after home births in Tanzania. Int J Gynecol Obstet. 2005;90:51-55.

24. Ripley DP, Burgess RW. External abdominal aortic compression: a study of a resuscitation manoeuvre for postpartum haemorrhage. Anaesth Intens Care. 1994;22:571575.

25. Akhter S, Begum MR, Kabir Z, Rashid M, Laila TR, Zabeen F. Use of a condom to control massive postpartum hemorrhage. Medscape General Medicine. 2003;5(3):38

26. Tsu VD. New and underused technologies to reduce maternal mortality. Lancet. 2003; 363:75-76.

27. Graham WJ, Bell JS, Bullough CW. Can skilled attendance at delivery reduce maternal mortality in developing countries? In: De Brouwere V, Van Lerberghe W, editors. Safe motherhood strategies: A review of the evidence. Studies in Health Organisational Services and Policy. 2001. p. 97-129.

28. World Health Organization. Skilled attendant at birth, 2005 estimates. Geneva (Switzerland). WHO; 2005.

29. Thaddeus S, Nangalia R. Perceptions matter: barriers to treatment of postpartum hemorrhage. J Midwifery Women's Health. 2004;49(4):293-297.

30. Cruz JR. Reduction of maternal mortality: the need for voluntary blood donors. Int J Gynecol Obsteet. 2007;98:291293.

John Snelgrove (MD 2011) is a medical student at the University of Western Ontario. He previously studied at McMaster University in the Bachelor of Health Sciences program and holds a MSc in social epidemiology from University College London in the U.K. 